\title{
Muscle nuclear changes in fetuses at risk for Duchenne muscular dystrophy
}

\author{
DEMETRIS VASSILOPOULOS AND ALAN E. H. EMERY
}

From the University Department of Human Genetics, Western General Hospital, Edinburgh

SUMMARY Muscle nuclear size was found to be significantly greater in fetuses at risk for Duchenne muscular dystrophy than in normal male fetuses of comparable gestational age. This supports the contention that the disease is already manifest in utero by the second trimester of pregnancy.

The muscle lesions in X-linked Duchenne muscular dystrophy (DMD), including preclinical cases, are well documented (Pearson, 1962; Hudgson et al., 1967; Bradley et al., 1972). Toop and Emery (1974) have also reported changes in muscle histology in fetuses at risk for DMD, and Webb (1974) suggested that muscle cell death in early fetal life could explain the pathogenesis of this disease.

Recently Vassilopoulos et al. (1976) reported a significant increase in muscle nuclear size in cases of DMD. This was explained as being probably a reflection of changes in nucleocytoplasmic relations. The present study was undertaken to see whether similar changes are also present in fetal dystrophic muscle.

\section{Materials and methods}

Muscle tissue from eight male fetuses at risk for DMD was examined. In four cases (Nos. 979, 1018,

Received for publication 22 March 1976

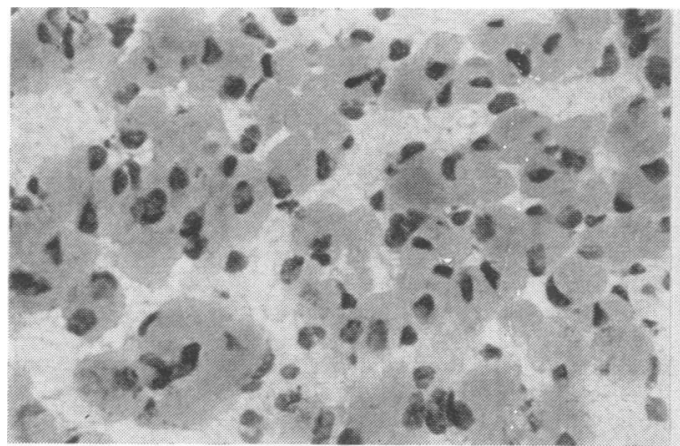

$75 / 479$, and $76 / 60$ ) the mothers were definite carriers and in the remaining four the mothers were possible carriers but at high risk (greater than 1 in 10) of having an affected son. In each case the sex of the fetus was established before abortion by sex chromatin and fluorescent studies on uncultured amniotic fluid cells and from karyotype analysis of cultured amniotic fluid cells. The results were compared with the findings in eight male fetuses of comparable gestational age obtained at abortion performed for social reasons and where there was no history of any neuromuscular disorder.

The gestational age of the fetuses was estimated by crown-rump (Hamilton and Boyd, 1962) and heeltoe (Streeter, 1921) measurements. Frozen sections $10-\mu$ thick stained with haematoxylin and eosin were examined (Figure). The cross-sectional areas of at least 100 nuclei closely apposed to the surface of transversely sectioned muscle fibres were estimated by planimetry at a final magnification of $\times 100$. The nuclei were selected for measurement in this way to

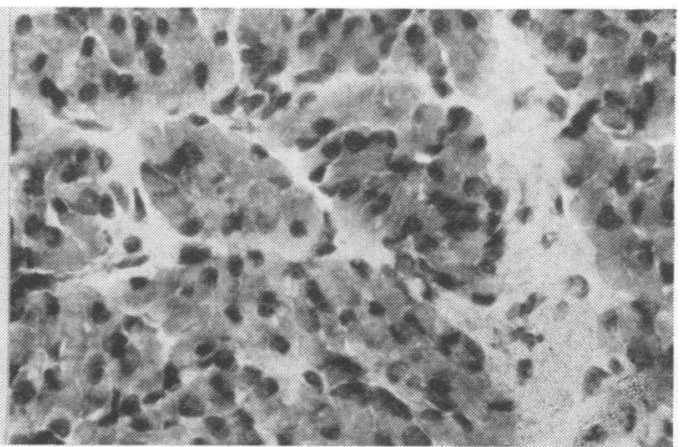

Fig. Transverse sections of muscle of fetus at risk for Duchenne muscular dystrophy (left) and of normal fetus of same gestational age (right). Cryostat sections. (Haematoxylin and eosin $\times 500$.) 
Table Mean ( \pm SD) muscle nuclear size in normal fetuses (controls) and in fetuses at risk for DMD

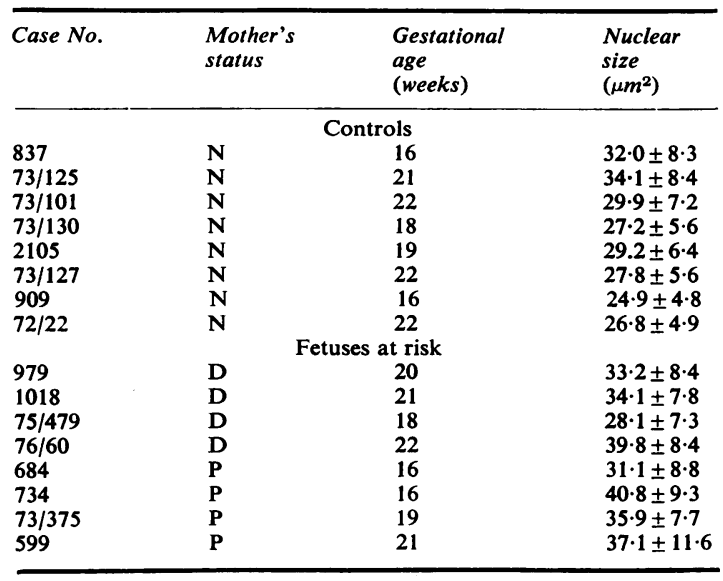

$\mathbf{N}=$ normal. $\quad \mathbf{D}=$ definite carrier. $\quad \mathbf{P}=$ possible carrier.

eliminate fibroblast, pericyte, endothelial cell, and satellite cell nuclei. Nuclear volume was not estimated since this requires certain assumptions to be made about the irregular shape of the muscle nucleus (Franke and Schinko, 1969). All measurements were made 'blind'-that is, without any knowledge of the source of the material.

\section{Results}

The results of the study are shown in the Table. Though there is overlap in the individual results obtained the overall mean size of nuclei in the group at risk for DMD $\left(35.0 \mu \mathrm{m}^{2} \pm 4 \cdot 2\right)$ is greater than in the matched controls $\left(28.9 \mu \mathrm{m}^{2} \pm 2 \cdot 9\right)$, the difference being statistically significant $(\mathrm{P}<0.002)$. A proportion of the fetuses at risk would be expected to be normal, but the results showed no evidence of bimodality and the lower values of fetuses at risk fell into the upper half of the control values. However, considering the high variability of the muscle fibre nuclear size it is difficult to draw any definite conclusions about this.

\section{Discussion}

Muscle weakness in Duchenne muscular dystrophy first becomes evident at about the age of 3 to 5 years. Pearson (1962), however, examined muscle tissue from a 2-month-old boy with preclinical Duchenne muscular dystrophy and found widespread hyalinization of the muscle fibres and an increased variation in their size. Bradley et al (1972), in a biopsy of muscle from a 17-day-old boy who later developed DMD, confirmed Pearson's findings and pointed out that pathological changes might well be evident even earlier. In a study of muscle histology in fetuses at risk for DMD, Toop and Emery (1974) in fact reported abnormalities similar to those described in preclinical cases and they concluded that certain histological changes were already evident in utero. In the present study the enlargement of muscle nuclei seen in fetuses at risk for DMD and the increase in muscle nuclear size in patients with DMD (Vassilopoulos et al., 1976) probably reflect the same underlying pathogenic process.

Nuclear changes in a variety of tissues have been reported in a number of diseases and experimental conditions but their significance is still controversial (Heiberg, 1957; Guimarães, 1971). The size of muscle fibre nuclei has been variously related to cellular hypertrophy (Doljanski, 1960; Goss, 1964), altered ionic environment (Davies and Spencer, 1962), and the degree of muscle contraction (Franke and Schinko, 1969). Enlargement of the nucleus seems to be one of the earliest and most consistent responses to alterations in nuclear environment.

Much information about nucleocytoplasmic relations has been gained from nuclear transplantation experiments in amphibia (Gurdon, 1968, 1970), and changes in nuclear size may well be related to alterations in gene expression (Lewin, 1974). This may explain the changes in muscle nuclear size in DMD and in fetuses at risk for DMD. In any event the findings reported in this study provide further support for Toop and Emery's (1974) suggestion that this disorder is already manifest in utero by the second trimester of pregnancy.

We thank all those who kindly supplied us with fetal material, particularly Drs R. Harris, J. B. S. Scrimgeour, and S. Walker. The study was supported by a grant from Muscular Dystrophy Group of Great Britain. D.V. is in receipt of a fellowship from the State Scholarship Foundation of Greece.

\section{References}

Bradley, W. G., Hudgson, P., Larson, P. F., Papapetropoulos, T. A., and Jenkison, M. (1972). Structural changes in the early stages of Duchenne muscular dystrophy. Journal of Neurology, Neurosurgery, and Psychiatry, 34, 451-455.

Davies, H. G., and Spencer, M. (1962). The variation in the structure of erythrocyte nuclei with fixation. Journal of Cell Biology, 14, 445-458.

Doljanski, F. (1960). The growth of the liver with special reference to mammals. International Review of Cytology, 10, 217-241.

Franke, W. M., and Schinko, W. (1969). Nuclear shape in muscle cells. Journal of Cell Biology, 42, 326-331.

Goss, R. I. (1964). Adaptive Growth. Logos Press, Academic Press, London.

Guimarães, R. C. (1971). The relationship between the nuclear volumes and the DNA contents of the hepatocyte nuclei in the normal liver, in the hepatosplenic form of 
Mansoni schistosomiasis and in hepatic cirrhosis. Revista Brasiliera de Pesquisas Medicas e Biologicas, 4, 169-183.

Gurdon, J. B. (1968). Changes in somatic cell nuclei inserted into growing and maturing amphibian öocytes. Journal of Embryology and Experimental Morphology, 20, 401-414.

Gurdon, J. B. (1970). Nuclear transplantation and the control of gene activity in animal development. Proceedings of the Royal Society of London, B176, 303-314.

Hamilton, W. J., and Boyd, H. W.(1962). Human Embryology, 4th ed. Helter and Sons, Cambridge.

Heiberg, K. A. (1957). Über die Erklärung einer Verschiedenheit der Krebszellen von anderen zellen. Nordisk Medicinhistorisk Arsbok, 8, 1-20.

Hudgson, P., Pearce, G. W., and Walton, J. N. (1967). Preclinical muscular dystrophy. Histopathological changes observed on muscle biopsy. Brain, 90, 565-576.
Lewin, B. (1974). Gene Expression, Vol. 2. Eucaryotic Chromosomes. J. Wiley, London.

Pearson, C. M. (1962). Histopathological features of muscle in the preclinical stage of muscular dystrophy. Brain, 85 , 109-120.

Streeter, G. L. (1921). Weight, sitting height, head size, foot length, and menstrual age of the human embryo. Contributions to Embryology, 11, 143-170.

Toop, J., and Emery, A. E. H. (1974). Muscle histology in fetuses at risk for Duchenne muscular dystrophy. Clinical Genetics, 5, 230-233.

Vassilopoulos, D., Lumb, E. M., and Emery, A. E. H. (1976). Muscle nuclear size in neuromuscular disease. Journal of Neurology, Neurosurgery, and Psychiatry, 39, 159-162.

Webb, J. N. (1974). Muscular dystrophy and muscle cell death in normal development. Nature, 252, 233-234. 